\section{AB0239 EFFECTS OF DYSMETABOLISMS AND COMORBIDITIES ON THE EFFICACY, SAFETY AND RETENTION RATE OF BIOLOGICAL DMARDS (BDMARD) IN INFLAMMATORY JOINT DISEASES.}

L. Cometi ${ }^{1}$, C. Bruni ${ }^{1}$, N. Chiti ${ }^{1}$, L. Tofani ${ }^{1}$, F. Nacci ${ }^{1}$, F. Bartoli ${ }^{2}$, S. Bellando Randone ${ }^{1,2}$, G. Fiori ${ }^{2}$, S. Guiducci ${ }^{1,2}$, M. Matucci-Cerinic ${ }^{1,2} .{ }^{1}$ University of Florence, Faculty of Medicine, Experimental and Clinical Medicine, Division of Rheumatology, Firenze, Italy; ${ }^{2}$ Careggi University Hospital, Rheumatology, Firenze, Italy

Background: bDMARDs have an effect on glucose homeostasis (1), lipoproteins profile $(2 ; 3)$ and blood pressure (4). However, with the exception of obesity $(5$; 6 ), there are no clear data on how bDMARDs work in patients who already have or develop metabolic comorbidities and whether these conditions can impact on their efficacy and safety profile.

Objectives: to evaluate, in chronic inflammatory joint diseases, the effect of arterial hypertension (AH), dyslipidemia (DYS) and diabetes mellitus (DM) on efficacy, safety and retention rate of first-line bDMARDs therapy.

Methods: a retrospective observational study on the clinical charts of Rheumatoid Arthritis (RA), Psoriatic Arthritis (PsA) or Ankylosing Spondylitis (AS), treated with first on-label bDMARD was performed. Data on adverse events, efficacy and comorbidities at the baseline visit in which the bDMARD was prescribed (BL), the visit performed after 6 months of therapy (6M), and the last visit on treatment (LoT) were collected.

Results: 383 patients $(41,8 \%$ RA, $33,4 \%$ PsA and $24,8 \%$ AS) were included in the study, with the predominance of females (F: $67,36 \%, M: 32,64 \%$; mean age $51,67 \pm$ 15,11 years). Our data show that the presence of comorbidities had no influence on efficacy of bDMARD, while patients who had DYS at BL manifested a higher rate of systemic adverse events either in the first 6 months of therapy (58,9\% vs $43,7 \%$, $\mathrm{p}=0,040)$ and also later on $(80,36 \%$ vs. $66,67 \%, p=0,046)$. In addition, patients who developed DYS and $\mathrm{AH}$ after the $6 \mathrm{M}$ visit reported a higher rate of systemic adverse events at LoT visit, compared to others (DYS: $97,8 \%$ vs $66,7 \%, p<0,001$; AH: $86,9 \%$ vs $65,2 \%, p=0,031$ ). For what concerns the retention rate, patients who developed DYS or AH during bDMARD treatment continued the drug for a longer period of time (DYS 95,5 vs 19,6 months, $p<0,001$; AH 72, 1 vs 23,4 months, $p<0,001)$. In particular, patients with $\mathrm{AH}$ who concomitantly carried out therapy with ACE-inhibitors (ACEi) and/or angiotensin II receptor blockers (ARB) continued bDMARDs for nearly 20 more months than patients who were not exposed to these drugs ( 40,5 vs 23,4 months, $p=0,001)$ and more frequently maintained the bDMARDS at LoT $(59,42 \%$ vs. $47,53 \%)$. In case of withdrawal in the ACEi/ARB exposed cohort, this was due to well-being and disease remission rather than inefficacy or adverse reaction $(p=0,025)$. In dyslipidemic patients treated with statins, data showed that bDMARDs were continued for a longer time than in DYS patients treated with other anti dyslipidemic therapies ( 41,09 vs. 26,50 months, $p=0,042)$. Conclusion: our data suggest that AH and DYS may be associated with higher frequency of adverse events but a better drug retention. The combination of bDMARD and ACEi/ARB may determine a better control of the inflammatory process by inhibition of angiotensin II, favouring the achievement of remission. In AH patients on bDMARDs, ACEi and ARB could therefore represent an useful anti-hypertensive drug choice. Similarly, statins could be the treatment of choice in DYS patients.

References:

[1] Gonzalez-Gay MA, et al. Clin Exp Rheumatol. 2006.

[2] Pollono EN, et al. Clin Rheumatol. 2010

[3] van Sijl AM, et al. Semin Arthritis Rheum. 2011.

[4] Yoshida S, et al. J Hum Hypertens. 2014.

[5] Gremese E, et al. Arthritis Care Res (Hoboken). 2013.

[6] Heimans L, et al. Arthritis Care Res (Hoboken). 2013.

Disclosure of Interests: Laura Cometi: None declared, Cosimo Bruni Speakers bureau: Actelion, Eli Lilly, Nicolò Chiti: None declared, Lorenzo Tofani: None declared, Francesca Nacci: None declared, Francesca Bartoli: None declared, Silvia Bellando Randone: None declared, Ginevra Fiori: None declared, Serena Guiducci: None declared, Marco Matucci-Cerinic Grant/research support from: Actelion, MSD, Bristol-Myers Squibb, Speakers bureau: Acetelion, Lilly, Boehringer Ingelheim

DOI: 10.1136/annrheumdis-2020-eular.2198

\section{AB0240 EXAMINING THE RELATIONSHIP BETWEEN RHEUMATOID ARTHRITIS, MULTIMORBIDITY AND ADVERSE HEALTH-RELATED OUTCOMES: A SYSTEMATIC REVIEW}

J. Canning ${ }^{1}$, S. Siebert ${ }^{2}$, B. Jani ${ }^{1}$, F. Mair ${ }^{1}$, B. Nicholl ${ }^{1}{ }^{1}$ University of Glasgow, General Practice and Primary Care, Glasgow, United Kingdom; ${ }^{2}$ University of Glasgow, Institute of Infection, Immunity and Inflammation, Glasgow, United Kingdom
Background: Rheumatoid arthritis (RA) is a chronic autoimmune disorder characterised by inflammation of the synovial joints causing pain, swelling and stiffness. Multimorbidity (the presence of two or more long-term conditions) affects approximately two thirds of people with RA. However, the relationship between RA and multimorbidity is poorly understand, as is the effect of this relationship on mortality and other health-related outcomes, particularly those relating to physical functioning and well-being.

Objectives: To explore existing literature to determine what is known about the effect, if any, of multimorbidity on mortality and other health-related outcomes in people with RA.

Methods: A systematic review was conducted following a protocol prepared using PRISMA-P 2015 reporting guidelines, ensuring the quality of the review. Studies were sourced from electronic medical databases, specifically MEDLINE, Embase, CINAHL, PsycINFO, The Cochrane Library and Scopus, using a pre-defined search strategy. Studies were selected based on specified eligibility criteria and quality appraised using the Cochrane Prognosis Methods Group-developed, Quality in Prognostic Studies (QUIPS) tool. A narrative synthesis of findings was conducted. Results: In total, 15 studies fulfilled our criteria for inclusion in our review. Of these, 7 studies had mortality as an outcome, with 6 reporting a significant association between multimorbidity and increased risk of all-cause mortality in people with RA. Nine studies had functional status/disability as an outcome, with 2 of these studies also including quality of life. All 9 studies reported significant associations between multimorbidity and the aforementioned health-related outcomes, demonstrating poorer functional status/increased disability and reduced quality of life in people with RA and multimorbidity.

Conclusion: Multimorbidity in people with RA is significantly associated with increased mortality and poor health-related outcomes in current literature. A better understanding of this relationship will provide an important foundation of knowledge to guide future health service design.

Acknowledgments: This work was supported by the Medical Research Council (MRC) [Grant Reference: MR/N013166/1]

Disclosure of Interests: Jordan Canning: None declared, Stefan Siebert Grant research support from: BMS, Boehringer Ingelheim, Celgene, GlaxoSmithKline, Janssen, Novartis, Pfizer, UCB, Consultant of: AbbVie, Boehringer Ingelheim, Janssen, Novartis, Pfizer, UCB, Speakers bureau: AbbVie, Celgene, Janssen, Novartis, Bhautesh Jani: None declared, Frances Mair: None declared, Barbara Nicholl: None declared

DOI: 10.1136/annrheumdis-2020-eular.4464

\section{AB0241 PREVALENCE OF ANXIOUS SYMPTOMS AND DEPRESSION IN A SAMPLE OF PATIENTS WITH RHEUMATOID ARTHRITIS (RA) AND OTHER CHRONIC RHEUMATIC DISEASES}

G. Crepaldi ${ }^{1}$, M. Voci ${ }^{1,2}$, M. Saracco ${ }^{1}$, A. Laezza ${ }^{2}$, P. Santino ${ }^{1}$, M. Marcato ${ }^{3}$, G. Rovera ${ }^{1,4}$, C. Lomater ${ }^{1} .{ }^{1}$ Rheumatology Unit, Ospedale Mauriziano, Turin, Italy; ${ }^{2}$ Clinical Phsycology Unit, Ospedale Mauriziano, Turin, Italy; ${ }^{3}$ Pharmacy Unit, Ospedale Mauriziano, Turin, Italy; ${ }^{4}$ Rheumatology Unit, Ospedale Sant'Andrea, Vercelli, Italy

Background: Clinical practice with patients suffering from chronic diseases highlights the presence of psychological symptoms of discomfort fed by biological and non-biological mechanisms linked to disease and treatment. In rheumatic diseases, literature detects the presence of anxious symptoms and depressed mood of clinical and sub-clinical importance with a multifactorial genesis'.

Objectives: To detect the impact on the state of health of anxious symptoms and depressed mood in a population suffering from RA and other rheumatic diseases in order to implement the effectiveness of psychological intervention through the selection of patients who present critical levels of discomfort.

Methods: Patients afferent to the Rheumatology outpatient clinic of Mauriziano Hospital have been screened from May 2018 to July 2018 with two self-administered questionnaires: HADS-A and HADS-D (Hospital Anxiety and Depression Scale), specifically developed for the evaluation of anxious and depressive symptoms in medical pathologies, and HAQ (Health Assessment Questionnaire) to explore functional disability. Data about rheumatic diagnosis and socio-demographic characteristics were also collected. Data were analyzed with descriptive statistics; the Student Test and the ANOVA test were used to evaluate prevalence and to compare the presentation of symptoms in the different diseases and the Pearson correlation coefficient was used to evaluate the relationship between symptoms and disability.

Results: A total of 427 subjects were screened (317 females and 110 males), aged between 19 and 90 years (mean $60 \pm 14 \mathrm{yrs}$ ). 156 subjects $(36.5 \%$ ) had a diagnosis of RA, $76(17.8 \%)$ of psoriatic arthritis, $42(9.8 \%)$ of ankylosing spondylitis, $14(3.3 \%)$ of systemic lupus erythematosus and $139(32.6 \%)$ of other rheumatic diseases (including Sjogren, osteoarthritis, fibromyalgia).

A high prevalence of anxious symptoms and depressed mood has been found and the number of subjects reporting scores indicating a clinically relevant 